\title{
Feasibility of Virtual Traffic Lights in Non-Line-Of-Sight Environments
}

\author{
Till Neudecker \\ Karlsruhe Institute of \\ Technology, Germany \\ till.neudecker@student.kit.edu \\ Tristan Gaugel \\ Karlsruhe Institute of \\ Technology, Germany \\ tristan.gaugel@kit.edu
}

\author{
Natalya An \\ Karlsruhe Institute of \\ Technology, Germany \\ an@kit.edu
}

\author{
Ozan K. Tonguz \\ Carnegie Mellon University \\ Pittsburgh, PA, USA \\ tonguz@ece.cmu.edu
}

\author{
Jens Mittag \\ Karlsruhe Institute of \\ Technology, Germany \\ jens.mittag@kit.edu
}

\begin{abstract}
Motivated by the idea to reduce deployment costs and to dynamically regulate vehicular traffic flows at intersections, inter-vehicle communications based virtual traffic lights are envisioned to replace traditional infrastructure based traffic lights. According to recent studies, virtual traffic lights are expected to increase traffic flow by up to $60 \%$. Yet, those studies were based on the assumption of a perfectly reliable communication, i.e., notification messages which signal a traffic light were always received by vehicles located within a certain distance to the sender. Hence, effects such as signal fading or non-line-of-sight conditions due to buildings were neglected. Such effects, however, can have a negative impact on the dissemination of the notification messages. This poster paper therefore studies whether these effects lead to significantly larger dissemination delays or not, and whether this increase is crucial for the feasibility of virtual traffic lights. According to the results of this study, the delay is not significantly larger, and virtual traffic lights seem to be feasible under such challenging conditions.
\end{abstract}

\section{Categories and Subject Descriptors}

C.2.1 [Computer-Communication Networks]: Network Architecture and Design-Wireless Communication

\section{Keywords}

Vehicular Networks, Traffic Applications, Traffic Lights

\section{INTRODUCTION}

Various inter-vehicle communications based traffic telematics applications are envisioned to improve the safety level and the efficiency of road traffic. While active safety applications aim to warn drivers of imminent dangers within their close vicinity, traffic efficiency applications intend to improve the traffic flow on a much larger scale, hence efficiency applications tolerate significantly larger information dissemination delays than safety applications.

In most of the envisioned applications, there is a clear relationship between the objective of the application and

Copyright is held by the author/owner(s).

VANET'12, June 25, 2012, Low Wood Bay, Lake District, UK. ACM 978-1-4503-1317-9/12/06. its requirements. However, there is one example which on the one hand aims to improve traffic efficiency, but on the other hand suffers from the same time-critical requirements as safety applications: dynamic intersection traffic management based on virtual traffic lights (VTL). Instead of regulating road traffic using traditional traffic lights with a fixed signaling schedule, virtual traffic lights are implemented in a distributed manner by vehicles themselves, and allow to self-adapt the signaling schedule with respect to the current traffic volume. While the introduction of virtual traffic lights reduces the cost of intersection management since no dedicated infrastructure has to be deployed, their usage may add a new safety risk if a robust and reliable operation is not guaranteed. For instance, if vehicles do not agree on one traffic signaling schedule, or if individual vehicles do not receive red light notifications early enough, conflicting and possibly dangerous driving maneuvers may be the consequence. Hence, VTL should only be implemented if a controlled and reliable operation can be ensured.

Recently, authors of [1] and [3] presented a decentralized approach to virtual traffic lights. In their two step approach, vehicles first have to agree on a virtual traffic light leader whenever they approach an intersection. This leader then adopts the role of a temporary virtual infrastructure, and broadcasts the traffic light schedule to neighboring vehicles. Eventually, this leader hands over the leadership to another vehicle and passes the intersection once it receives a green light notification. According to the results presented by the authors, this approach is able to increase traffic flow in urban areas by up to $60 \%$ in comparison to traditional intersection management. However, their evaluation is based on optimistic and ideal assumptions, i.e., they neglected the existence of radio obstacles, and considered a perfect communications system based on which every broadcast message is received by every vehicle within a certain distance.

Apparently, the assumptions made in [1] are not realistic. For instance, as measurement campaigns have shown, e.g., in [2], non-line-of-sight (NLOS) conditions do exist at most intersections in urban or suburban environments. Hence, a perfect and reliable delivery of messages "around the corner" can not always be assumed. Further, concurrent packet transmissions by multiple vehicles might further reduce the communication performance due to resulting packet collisions. As a consequence, one can not rely on the successful 
reception of every single message, which can lead to the situation that either the election process does not succeed in time, or traffic signaling messages are not received in time.

In this poster paper we therefore study the feasibility of a virtual traffic light application under NLOS conditions, i.e., in urban and suburban environments. In particular, we provide an answer to the question whether NLOS conditions and signal fading prohibit a timely election of the virtual traffic light leader.

\section{VIRTUAL TRAFFIC LIGHTS}

In this section we first briefly describe the general concept of VTL as presented by Ferreira et al. (refer to the original paper in [1] for more details). Then, we discuss the impact of realistic communication effects such as radio obstacles and signal fading.

\section{General Concept}

According to [1], the overall process of VTL is divided into two phases: Election of a VTL Leader and Handover of VTL Leadership. In order to support the VTL application, in particular the first stage, all vehicles are assumed to periodically broadcast Content Awareness Messages (CAM). Such messages contain at least the own position and driving direction, such that each vehicle is able to establish mutual awareness. Further, the own VTL status is included.

For the election of a VTL leader possible candidates (denoted as cluster leaders) are determined for each road segment that leads towards the intersection. The cluster leader is defined as the vehicle which is closest to the intersection (in comparison to all other vehicles in the same cluster). Each cluster leader is a candidate for the VTL leader role and follows the same rules to decide whether it is going to be the VTL leader or not. Namely, a cluster leader ensures that at least one more conflicting vehicle is approaching the intersection and checks that the conflicting cluster leader is closer to the intersection, so that the vehicles that are already closer to the intersection get a green light in order to avoid hard braking or a possible accident. If above conditions are fulfilled for one of the candidates, it adopts the VTL leader role, and takes control. During the control period, the VTL leader schedules the flow of all other clusters, and changes the traffic light signals if either no vehicles remain to be scheduled, or if the time slice has expired. In the latter case, VTL leader needs to handover its VTL leadership. During the handover the current VTL leader selects a new leader out of the existing cluster leaders. Shortly before handing over the control, the current VTL leader assigns a green signal to its own cluster to continue his travel, and a red signal to the cluster of the new VTL leader.

\section{Impact of Realistic Communication Effects}

As already stated, fading channel and radio obstacles may influence the performance and feasibility of a virtual traffic light system. While it is usually not safety-critical if vehicles in a cluster initially disagree on the current cluster leader, it is critical if VTL leadership is adopted too late or not at all as vehicles would be unable to react accordingly. In the following we will thus only focus on the VTL leader election itself, as the corresponding communication conditions are generally worse, compared to handovers or traffic light signal distribution (where occasionally even line-of-sight can be assumed).

\section{EVALUATION}

As the focus of this work is not the evaluation of large scale traffic efficiency improvements by VTL, but instead to investigate whether VTL is feasible under NLOS conditions, we limit the scope of the evaluation to a scenario with only one intersection. An analytical approach is used to assess worst case conditions, whereby a simulation based evaluation, with a more realistic modeling of protocol and driver behavior, allowed us to stress different aspects of VTL.

For our scenario we took a typical representative city intersections. It consists of a regular, single lane, 4-way intersection with an inter-building distance of $22 \mathrm{~m}$. Radio propagation was modeled using the VirtualSource11p NLOS communication model [2]. Note that the difference between the urban and suburban ${ }^{1}$ configuration for the radio propagation model is an additional path loss of $2.94 \mathrm{~dB}$ with suburban settings. The evaluation itself is based on the following metrics:

- VTL leader election distance $[m]$ : the distance at which the coordination of the VTL protocol is finished and a vehicle declares itself as the VTL leader. Consequently, it is a lower bound distance of when a driver is signalized to stop.

- Required deceleration $\left[\mathrm{m} / \mathrm{s}^{2}\right]$ : This metric can be directly deduced from the distance at which a driver is signalized to stop and the vehicle's speed. It indicates the minimum deceleration value a driver has to achieve on average in order to be able to stop before the intersection. It can be viewed as one indicator of the safety state a driver is in.

\subsection{Results}

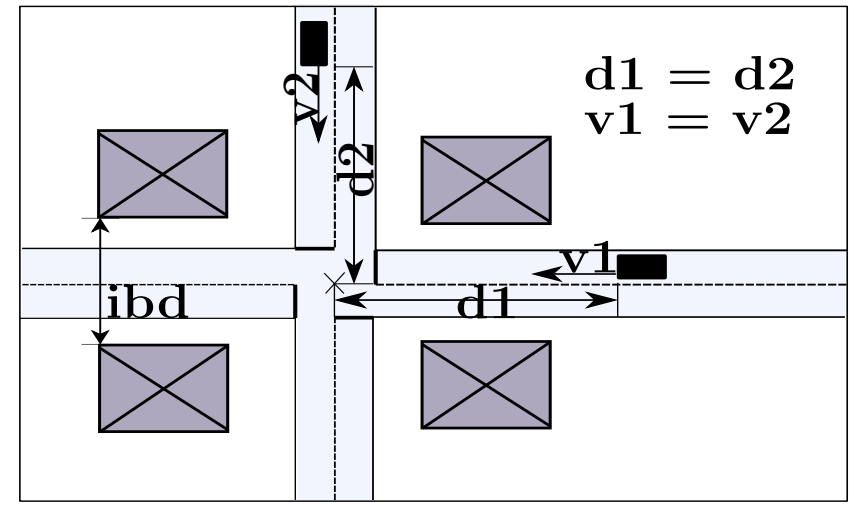

Figure 1: The worst case scenario layout. Speed and distance to the intersection crossing of both vehicles are exactly the same. Inter-building distance (ibd) is equal to $22 \mathrm{~m}$.

In the following, we present an analytical evaluation of the worst case scenario. This scenario consists of two vehicles on perpendicular roads that are simultaneously approaching an empty intersection, as depicted in Figure 1. In this setup

\footnotetext{
$1_{\text {The NLOS communication model considers suburban environments }}$ to be similar to urban environments with the addition of plants and bushes in front of the buildings. In this sense, suburban environments correspond to worse RF propagation conditions than in urban environments.
} 


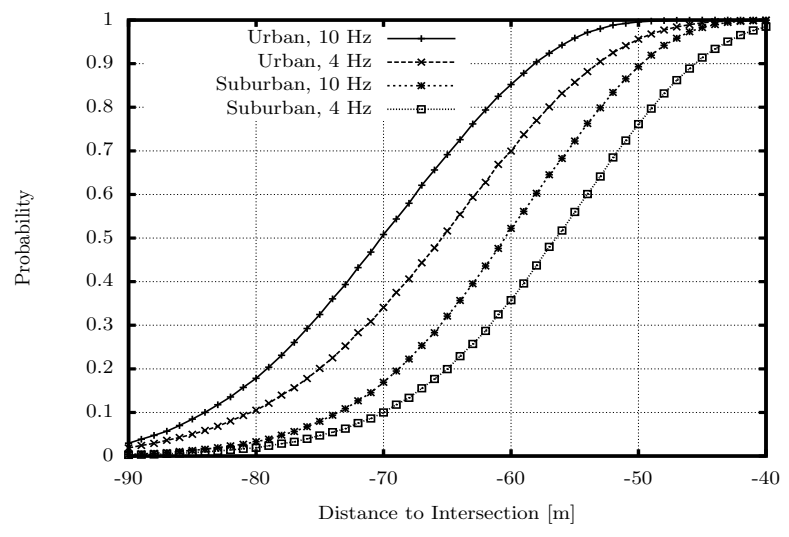

Figure 2: Probability of receiving at least one message from a vehicle on a perpendicular road, equally far away from the intersection, until distance $\mathrm{x}$

no VTL leader is already existent and the communication conditions are worst due to both vehicles being equally far away from the intersection. Since both vehicles are respectively their own cluster leaders, a vehicle becomes aware of its status as being the designated VTL leader the first time a CAM message from another vehicle is received. Figure 2 depicts the probability that at a certain distance to the intersection at least one CAM message was received. We are hereby considering packet generation rates of $4 \mathrm{~Hz}$ and $10 \mathrm{~Hz}$ (corresponding to beacon messages every $250 \mathrm{~ms}$ and $100 \mathrm{~ms}$, respectively), as well as urban and suburban communication conditions. It can be clearly seen, that under the condition of synchronously approaching vehicles i) no communication at distances of more than 90 meters from the intersection is reasonable; and that ii) at a distance of 40 meters there is a high probability that a vehicle is aware of its status as being the VTL leader.

The distance at which a vehicle becomes aware of its status as VTL leader is equivalent to the distance at which the corresponding driver is signalized to stop. Hence, one can calculate the required deceleration of the driver in order to be able to stop before the intersection. The required deceleration of the VTL leader is the maximum over all vehicles, since it is the closest vehicle to the intersection. In Figure 3 we plot the resulting required deceleration for various communication settings. As can be seen, the required deceleration decreases rapidly for every setting and even in worst case considered communication conditions reaches zero with non critical values and does not exceed $-3 \mathrm{~m} / \mathrm{s}^{2}$.

The analytical assessment is affirmed by our simulation results, with better overall results. Due to space restrictions, however, we are only able to briefly outline some simulation based results.

- The probability of a vehicle being aware of its status as designated VTL leader at a certain distance is in general higher. This is due to the fact that, contrary to the analytical worst case assumption, cluster leaders are in general not simultaneously approaching an intersection. As an example: The probability of being aware of the VTL leader status is increased from about $52 \%$ (Urban, $4 \mathrm{~Hz}$ ) at a distance of 65 meters (see Figure 2 ) to $89 \%$ over all simulated scenarios.

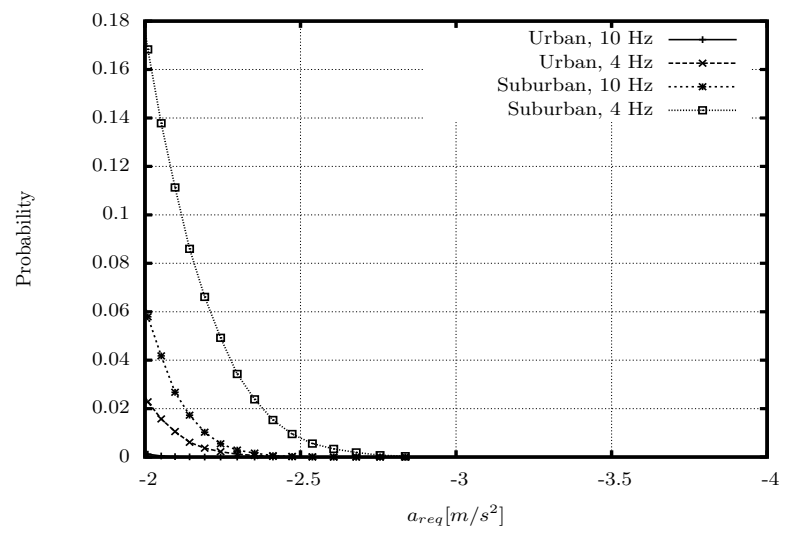

Figure 3: Minimum required deceleration values the designated VTL leader has to achieve in order to be able to stop in front of the intersection

- On average, 16 out of 100 simulated vehicles become a VTL leader, whereby only 6 "regular" leader elections take place and 10 handovers are performed. Handovers are in general less critical than regular elections, since i) the old VTL leader is in close vicinity to the intersection and ii) even line-of-sight conditions can be assumed for several seconds while the old VTL leader is at the center of the intersection.

\section{SUMMARY}

In this paper we investigated the feasibility of virtual traffic lights in a basic intersection scenario that exhibits NLOS conditions. While previous studies have shown that the VTL concept works and is feasible in a "perfect world", NLOS channel conditions, as they exist in the "real world", may lead to a different outcome of the assessment.

According to the results shown in this paper, the expected impact of NLOS conditions on the performance of VTL can be confirmed. However, the results indicate that this impact is not significant and that NLOS conditions do not prohibit a timely detection and the possibility to take appropriate actions. In particular, the results show that the traffic flow regulation starts early enough so that all vehicles become aware of the current traffic signal schedule in time. Hence, each vehicle is able to react and adhere to the instructions given by the VTL leader. While these results show the feasibility of Virtual Traffic Lights under NLOS channel conditions, further research is needed to cover all the possible scenarios and other sources of impairment.

\section{REFERENCES}

[1] M. Ferreira, R. Fernandes, H. Conceicão, W. Viriyasitavat, and O. K. Tonguz. Self-organized Traffic Control. In the Proceedings of the seventh ACM international workshop on VehiculAr InterNETworking, VANET '10, pages 85-90, New York, NY, USA, 2010. ACM.

[2] T. Mangel, O. Klemp, and H. Hartenstein. A Validated $5.9 \mathrm{GHz}$ Non-Line-Of-Sight Path-Loss and Fading Model for Inter-Vehicle Communication. In the Proceedings of the 11th International Conference on Telecommunications for Intelligent Transport Systems, pages 75-80, August 2011.

[3] O. K. Tonguz. Biologically inspired solutions to fundamental transportation problems. IEEE Communications Magazine, 49(11):106 -115, November 2011. 